\title{
Avaliação do Processo de Medição para Serviços de TI em uma Empresa Global à Luz do MR-MPS-SV
}

\author{
Bianca Trinkenreich, Gleison Santos
}

Programa de Pós-Graduação em Informática, Departamento de Informática Aplicada, Universidade Federal do Estado do Rio de Janeiro (UNIRIO), Av. Pasteur, RJ, Brasil

\{bianca.trinkenreich, gleison.santos\} @uniriotec.br

\begin{abstract}
An effective measurement process helps organizations to understand their capabilities to develop workable plans to execute and deliver agreed services to customers. MR-MPS-SV measurement process supports organizations on understanding processes behavior and therefore also supports decision making to control and improve them. We present an experience report in a large global company to evaluate adherence to the MR-MPS-SV Measurement process on measuring processes Incident Management, Service Continuity and Availability Management, and Budget and Accounting Service. As a result, we were able to identify improvement opportunities for both measurement process and measures in use aiming to provide better alignment to business goals. We found process mapping highlighting integration between different processes is a good practice to include measures related to more than one process. Also, performance measures spread for several teams promote teamwork and mutual support between different teams.
\end{abstract}

Resumo. Um processo de medição eficaz ajuda a compreensão da habilidade de uma organização em desenvolver planos viáveis para executar e entregar os serviços acordados com seus clientes. No MR-MPS-SV, o processo Medição apoia a compreensão do comportamento dos processos e, com isso, a tomada de decisão para controlá-los e melhorá-los. Este artigo apresenta um relato de experiência sobre a medição nos processos de Gerência de Incidentes, Gerência de Continuidade e Disponibilidade e Orçamento e Contabilização de Serviços de uma empresa global de grande porte, visando avaliar a aderência do processo adotado à luz do processo Medição do modelo de maturidade MRMPS-SV. Como resultado, foram identificadas oportunidades de melhoria para o processo de medição e para as próprias medidas para atenderem melhor os objetivos da empresa. Também foi observado que o mapeamento das integrações entre os processos para inclusão de medidas correlacionando mais de um processo pode ser realizado para apoiar a seleção de medidas, e que o uso de medidas de desempenho permeando diferentes equipes estimulam o trabalho e o apoio entre diferentes equipes.

\section{Introdução}

Orientação sobre como desenvolver e melhorar as práticas de maturidade de serviços é um fator chave para o desempenho do fornecedor de serviços e a satisfação do cliente. Os modelos CMMI-SVC [Forrester et al., 2010] e MR-MPS-SV [Softex, 2012a] foram

TRINKENREICH, B.; SANTOS, G.

Avaliação do Processo de Medição para Serviços de TI em uma Empresa Global à Luz do MR-MPS-SV

iSys - Revista Brasileira de Sistemas de Informação, Rio de Janeiro, vol. 8, No. 2, p. 58-77, 2015 
criados para atender a esta necessidade. Em ambos os modelos, a medição é fundamental à tomada de decisões para melhoria de processos da organização.

O fato de ser capaz de fazer previsões em relação aos serviços que entrega é um fator de sucesso para a organização. Um processo de medição eficaz ajuda a empresa a compreender suas capacidades para que possa desenvolver planos viáveis para executar e entregar os serviços acordados com seus clientes. Medições também ajudam a detectar tendências e antecipar problemas, proporcionando assim um melhor controle de custos, redução de riscos, melhoria da qualidade e garantia de alcance dos objetivos de negócio [Florac e Carleton, 1997]. Além disso, quando realizada apropriadamente, a medição é fundamental em atividades de melhoria de processos.

Apesar de modelos de maturidade, normas, padrões e bibliotecas de boas práticas de serviços colocarem a medição como fundamental para gerenciamento de serviços de TI e citarem exemplos de possíveis medidas a serem utilizadas, uma dificuldade que as organizações prestadoras de serviços de TI apresentam é fazer a seleção das medidas mais apropriadas para atender os objetivos de negócio da empresa. A falta de uma abordagem estruturada para seleção de indicadores de serviços de TI e exemplos práticos são dois fatores que causam tal dificuldade, além do fato de as ferramentas em uso pelas equipes de suporte geralmente não incluírem a medição [Jäntti et al., 2010] [Lepmets et al., 2014].

Em [Trinkenreich et al., 2015] os autores apresentam um mapeamento sistemático objetivando encontrar medidas que podem ser utilizadas em iniciativas de modelos de maturidade de serviços de TI. Em [Trinkenreich e Santos, 2015] os resultados de [Trinkenreich et al., 2015] foram utilizados para avaliar a correlação entre as medidas e novas medidas de serviços em uso por uma organização. Este artigo apresenta um relato de experiência sobre a avaliação da medição realizada sobre os processos de Gerência de Incidentes, Gerência de Continuidade e Disponibilidade dos Serviços e Orçamento e Contabilização de Serviços de uma empresa global de grande porte. Além do processo de medição, foi analisado se as medidas em uso pela organização atendem os objetivos de negócio e também a estabilidade e a capacidade destas medidas em atender ao indicador colocado como meta anual pela organização.

A avaliação do processo atual foi guiada à luz dos resultados esperados do processo Medição do MS-MPS-SV e a avaliação das medidas foi guiada à luz de revisões na literatura. Neste trabalho mostramos a evolução de [Trinkenreich e Santos, 2014] que incluiu avaliação de medidas relacionadas aos processos Gerência de Incidentes e Gerência de Continuidade e Disponibilidade dos Serviços, incluindo a avaliação de mais uma medida, sobre o processo de Orçamento e Contabilização de Serviços. Este processo não teve nenhuma medida encontrada em [Trinkenreich et al., 2015] mas é sugerido para controle de custo efetivo de produção para controle financeiro por [Brooks, 2014].

Este artigo está composto da seguinte forma: a Seção 2 apresenta a revisão da literatura sobre Qualidade de Serviços abordando tópicos relacionados e este trabalho, a Seção 3 apresenta trabalhos relacionados anteriores dos autores, a Seção 4 apresenta a descrição da experiência e, por fim, a Seção 5 apresenta as considerações finais. 


\section{Fundamentação teórica}

\subsection{Qualidade de Serviços}

Gerenciamento de serviços de TI é um conjunto de habilidades organizacionais específicas capazes de fornecer valor aos clientes por meio de serviços. Sua prática tem crescido com a adoção de uma abordagem orientada aos serviços de gerenciamento de TI para suportar aplicações, infraestrutura e processos [OGC, 2011]. Qualidade do serviço é um conceito abstrato, devido à natureza do termo "serviço", que é intangível, não homogêneo e seu consumo e produção são inseparáveis [Parasuraman et al., 1985]. A fim de oferecer qualidade, o fornecedor deve avaliar continuamente o modo pelo qual o serviço está sendo fornecido e o que o seu cliente espera no futuro. Um cliente estará insatisfeito com seu prestador de serviços de TI que em alguns casos supera suas expectativas, mas em outros momentos não atende as expectativas e lhe decepciona.

Ao apresentar elementos essenciais de processos eficazes e um caminho evolutivo para a melhoria, os modelos de maturidade fornecem orientações sobre como estruturar os processos, como uma aplicação de princípios para atender um ciclo de melhoria contínua de processos [Forrester et al., 2010].

\subsubsection{Modelo de Maturidade de Serviços MR-MPS-SV}

O Modelo de Referência MPS para Serviços (MR-MPS-SV) [Softex, 2012a] é um modelo de maturidade voltado principalmente para micro, pequenas e médias empresas que fornecem serviços de TI e permite avaliar a execução dos processos com diferentes graus de efetividade. A definição dos processos se baseia nos seguintes modelos e normas: ISO/IEC 15504-2 [ISO/IEC, 2003], MR-MPS-SW [Softex, 2012b], ISO/IEC 20000 [ISO/IEC, 2011], CMMI-SVC [Forrester et al., 2010]. A Tabela 1 mostra os sete níveis de maturidade do modelo MR-MPS-SV [Softex, 2012a] (de G para A, o mais alto), composto por 24 processos, dos quais 12 são baseados no padrão de qualidade de serviços ISO/IEC 20000 e, portanto, não têm equivalente no MR-MPS-SW (em itálico na Tabela 1). A capacidade do processo é representada por um conjunto de Atributos de Processo (AP) descritos em termos de Resultados dos Atributos de Processos (RAPs), que estabelecem os resultados a serem atingidos com a implementação real do processo, podem ser demonstrados por um produto de trabalho elaborado ou uma mudança relevante de estado ao se executar o processo, e evoluem de acordo com o nível de maturidade. Quanto maior a maturidade, um maior nível de capacidade para desempenhar o processo deve ser atingido. A evolução e a obtenção de cada nível de maturidade ocorrem quando são atendidos os objetivos e todos os resultados esperados dos respectivos processos, além dos RAPs determinados para aquele nível [Softex, 2012a].

Tabela 1. Processos do Modelo de Maturidade de Serviços MR-MPS-SV (Softex, 2012a)

\begin{tabular}{|c|l|}
\hline Nível & \multicolumn{1}{|c|}{ Processos } \\
\hline $\mathrm{A}$ & (não há novos processos) \\
\hline $\mathrm{B}$ & Gerência de Trabalhos (GTR) (evolução) \\
\hline $\mathrm{C}$ & $\begin{array}{l}\text { Gerência de Decisões (GDE), Gerência de Riscos (GRI), Gerência de Capacidade (GCA), Gerência } \\
\text { de Continuidade e Disponibilidade de Serviços (GCD), Gerência de Liberações (GLI), Gerência de } \\
\text { Segurança de Informação (GSI), Gerência de Relato de Serviços (RLS) }\end{array}$ \\
\hline
\end{tabular}

TRINKENREICH, B.; SANTOS, G.

Avaliação do Processo de Medição para Serviços de TI em uma Empresa Global à Luz do MR-MPS-SV

iSys - Revista Brasileira de Sistemas de Informação, Rio de Janeiro, vol. 8, No. 2, p. 58-77, 2015 


\begin{tabular}{|c|l|}
\hline Nível & \multicolumn{1}{|c|}{ Processos } \\
\hline D & Desenvolvimento do Sistema de Serviços (DSS), Orçamento e Contabilização de Serviços (OCS) \\
\hline E & $\begin{array}{l}\text { Definição do Processo Organizacional (DFP), Avaliação e Melhoria do Processo Organizacional } \\
\text { (AMP), Gerência de Mudanças (GMU), Gerência de Recursos Humanos (GRH) e Gerência de } \\
\text { Trabalhos (GTR) (evolução) }\end{array}$ \\
\hline F & $\begin{array}{l}\text { Medição (MED), Aquisição (AQU), Gerência de Configuração (GCO), Garantia da Qualidade (GQA), } \\
\text { Gerência de Portfolio de Trabalhos (GPT), Gerência de Problemas (GPL) }\end{array}$ \\
\hline G & $\begin{array}{l}\text { Gerência de Requisitos (GRE), Gerência de Trabalhos (GTR), Entrega de Serviços (ETS), Gerência } \\
\text { de Incidentes (GIN), Gerência de Nivel de Serviço (GNS) }\end{array}$ \\
\hline
\end{tabular}

Os processos abordados neste artigo (Medição, Gerência de Incidentes, Gerência de Continuidade e Disponibilidade de Serviços e Orçamento e Contabilização de Serviços) serão apresentados a seguir.

Um incidente é "uma interrupção não planejada de um serviço, uma redução na qualidade de um serviço ou um evento que ainda não impactou o serviço para o cliente" [ISO/IEC, 2011]. O propósito do processo Gerência de Incidentes (GIN), previsto no nível G do MR-MPS-SV, é restaurar os serviços aos níveis acordados e cumprir as solicitações de acordo com os Acordos de Nível de Serviço (ANS) entre o fornecedor e seu cliente [Softex, 2012a]. Gerenciar inclui registrar, acompanhar, escalonar (se necessário), e encerrar o incidente. Quanto mais rapidamente um incidente é tratado, maior a disponibilidade do serviço e a satisfação do usuário. O prazo para atendimento dos incidentes deve ser alinhado com os objetivos do negócio e registrado no ANS. Desta forma, GIN se relaciona com o processo Gerência de Nível de Serviço (GNS), também parte do nível G do MR-MPS-SV, cujo objetivo é garantir o cumprimento do ANS [Softex, 2012a].

Um processo especial para tratar incidentes mais graves deve ser criado, definindo o que é um incidente grave, quem tem a autoridade para declará-lo desta forma, quem deve coordenar sua resolução, como ser resolvido, quais comunicações devem ser realizadas durante a resolução e qual o relacionamento com o processo Gerência de Continuidade e Disponibilidade dos Serviços (GCD), quando sua invocação é necessária [ISO/IEC, 2012]. O processo GCD, do nível C do MR-MPS-SV, tem como propósito assegurar que acordos de níveis de serviços sejam cumpridos em circunstâncias previsíveis, protegendo os interesses dos clientes e demais partes interessadas. Mesmo com altos níveis de disponibilidade, sempre existe a possibilidade de ocorrerem falhas. A gerência de disponibilidade garante que haja uma resposta profissional para situações indesejáveis.

O propósito do processo Orçamento e Contabilização de Serviços (OCS), presente no nível D do MR-MPS-SV, é controlar o orçamento e a contabilização dos serviços fornecidos. Este processo inclui as etapas de planejamento e controle de custos, estimando o custo do fornecimento do serviço, criando os orçamentos, controlando, solucionando e comunicando aos interessados sobre seus possíveis desvios. Os recursos necessários para a prestação do serviço de uma organização (equipamentos, licenças de software, pessoas, serviços fornecidos por terceiros etc.) devem ser orçados, contabilizados e posteriormente controlados [ISO/IEC, 2011]. A medição aparece como uma forma valiosa para apoiar o controle do orçamento. Se o objetivo estratégico da organização incluir metas de redução de custo neste sentido, podem ser utilizadas medidas para avaliar o quanto está sendo reduzido do orçamento. 
O processo Medição (MED) faz parte do nível F do MR-MPS-SV e inclui coleta, armazenamento, análise e relato sobre dados relativos aos serviços fornecidos e os processos realizados na organização. A medição fornece informações quantitativas para apoiar a tomada de decisão [Softex, 2012a]. É fundamental selecionar medidas adequadas para apoiar esta tomada de decisão, baseadas nos processos mais relevantes para os objetivos da organização, aqueles que podem contribuir para seu sucesso ou fracasso. Como não há direcionamento claro sobre que processos seriam relevantes, uma das dificuldades das organizações é selecionar processos e medidas adequados para monitorar e melhorar o gerenciamento da qualidade dos serviços fornecidos, afetando positivamente a percepção de qualidade dos clientes.

Todos os processos do MR-MPS-SV devem ser medidos (devido ao RAP 4, que descreve que as medidas devem ser planejadas e coletadas para monitorar a execução do processo e ajustes necessário devem ser realizados), mas não há uma indicação direta sobre quais aspectos relevantes devem ser selecionados como alvo. O guia de implementação sugere 8 exemplos, todos de processos específicos do MR-MPS-SV: Gerência de Configuração (GCO), Gerência de Incidentes (GIN), Gerência de Problemas (GPL), Gerência de Nível de Serviço (GNS), Gerência de Mudanças (GMU), Gerência de Liberação (GLI), Gerência de Continuidade e Disponibilidade de Serviço (GCD) e Gerência de Capacidade (GCA) [Softex, 2012c]. Não é sugerida nenhuma medida sobre o processo Orçamento e Contabilização de Serviços (OCS), que também é específico do MR-MPS-SV. A medição no MR-MPS-SV é realizada de várias formas, de acordo com o nível de maturidade. Nos níveis G a C do MR-MPS-SV, apesar de não ser a melhor prática, as medidas consideradas durante a implementação do processo Medição podem se limitar à comparação do executado com o planejado. No entanto, nos níveis mais altos de maturidade (ou seja, os níveis B e A), é necessário conhecer o comportamento do processo, aplicando técnicas de controle estatístico de processos, e prever se o desempenho e qualidade serão capazes de atender aos objetivos estabelecidos para alcançar a melhoria contínua dos processos [Rocha et al., 2012].

\subsubsection{Controle Estatístico de Processos}

O Controle Estatístico de Processos nasceu na manufatura, para analisar linhas de produção. Com gráficos de controle e métodos estatísticos, utiliza dados estáveis e coletados de execuções passadas de processos. Desta forma, fornece uma visão quantitativa sobre o desempenho destes processos em execuções correntes e futuras, identificando ações corretivas quando for aplicável [Rocha et al., 2012].

Em um processo estável as variações acontecem, mas são aceitáveis e controladas. Elas ocorrem dentro de limites previsíveis, esperados, tornando possível predizer seu comportamento. Um processo estável não necessariamente é capaz de alcançar o desempenho que o negócio espera dele. Aa ações corretivas devem ser tomadas para melhorar o desempenho do processo e trazê-lo à taxa acordada [Rocha et al., 2012].

A execução do Controle Estatístico de Processos não é feita para todos os processos de negócios da organização, apenas para subprocessos selecionados, os mais críticos que impactem os objetivos ou contribuam para o fracasso deles [Rocha et al., 2012]. Os principais critérios que devem apoiar esta decisão são a relação do subprocesso com os objetivos relevantes de negócio, a existência de dados a serem usados, o grau de variabilidade dos dados, a estabilidade do subprocesso e a possibilidade de modelos 
preditivos serem construídos a partir das informações disponíveis na organização [Forrester et al., 2010].

Os gráficos de controle são usados para mostrar variações no comportamento e analisar a estabilidade dos processos, diferenciando os sinais (causas especiais que devem ser investigadas) dos ruídos (causas comuns aceitáveis). O layout básico de um gráfico de controle possui limite superior e inferior distanciados de três desvios padrão da linha central. Os testes mais conhecidos de estabilidade são: algum ponto estiver fora dos limites, dois de três pontos do mesmo lado em relação à linha central e também a mais de dois desvios padrão de distância dela, quatro de cinco pontos sucessivos do mesmo lado em relação à linha central e também a mais de um desvio padrão de distância dela, e oito pontos sucessivos do mesmo lado em relação à linha central [Rocha et al., 2012].

\section{Trabalhos anteriores dos autores}

Em [Trinkenreich e Santos, 2014], os autores realizaram a avaliação do processo de medição de serviços em uma organização, contemplando medidas relacionadas aos processos de Gerência de Incidentes (GIN) e Gerência de Continuidade e Disponibilidade (GCD). A avaliação do processo de medição foi conduzida sob a luz dos resultados esperados do processo Medição (MED), parte do nível F do modelo de maturidade MRMPS-SV [Softex, 2012a]. Neste trabalho, os autores avaliaram se o processo de medição, independente das medidas consideradas, estava aderente ao modelo. Além disso, os autores avaliaram as próprias medidas sob dois aspectos: (i) se os processos são estáveis e capazes de atender as metas anuais da organização, sob a luz do Controle Estatístico de Processos [Rocha et al., 2012]; e (ii) se as medidas em uso pela organização são capazes de atender o objetivo do negócio, sob a luz do GQM [Solingen e Berghout, 1999]. Como resultado, são apresentadas as lições aprendidas com a experiência, e melhorias ao processo MED são sugeridas. Além disso, novas medidas a serem usadas pela organização, encontradas em revisões informais na literatura, com objetivo de atender mais diretamente o objetivo organizacional também foram apresentadas para serem avaliadas em relação à incorporação aos planos de medição.

Os autores, então, perceberam a necessidade de buscar na literatura quais medidas estão sendo usadas para melhoria da qualidade de serviços. Em [Trinkenreich et al., 2015], realizaram um estudo baseado em mapeamento sistemático buscando métricas adequadas para atender iniciativas de maturidade de processos de serviços de TI. Após leitura dos artigos selecionados pelos critérios de inclusão e exclusão definidos, os dados coletados foram resumidos, medidas semelhantes foram analisadas considerando o seu nome, descrição e fórmula (quando disponível) e classificadas por processo do MR-MPSSV [Softex, 2012a]. Durante a análise para classificação das medidas por processos, os autores constataram o que já havia sido percebido na revisão informal da literatura anterior: que existem medidas que não são relacionadas a somente um processo, mas a mais de um processo, e que tais relacionamentos podem ser de causa-efeito entre diferentes processos, e merecem estudos mais aprofundados. Como resultado, os autores geraram uma lista de medidas classificadas por processos do MR-MPS-SV [Softex, 2012a] para apoiar iniciativas de medição de qualidade de serviços e iniciativas de modelos de maturidade de serviços de TI.

Em seguida, foi realizado um estudo de caso na indústria [Trinkenreich e Santos, 2015] para verificar que tipo de relação existe entre os processos que aparecem em 
medidas que incluem mais de um processo e avaliar se as medidas em uso pela organização foram retornadas pelo mapeamento sistemático realizado em [Trinkenreich et al., 2015]. A primeira etapa realizada para a avaliação de causa-efeito entre as métricas foi de aumento ou redução entre pares de medidas. A segunda etapa realizada para a avaliação de causa-efeito entre as métricas foi, utilizando um exemplo de "Percentual de incidentes causados por mudanças", a aplicação de testes estatísticos de correlação (Pearson e Spearman) entre as mudanças executadas e os incidentes abertos. A correlação estatística não foi encontrada nos dados utilizados (totais para todas as aplicações de uma mesma infraestrutura), mas os gerentes da organização considerada informaram que conseguem notar erros e casos de indisponibilidade depois de algumas mudanças, que precisam ser investigados. Como resultado, novas medidas foram acrescentadas à lista obtida com o mapeamento sistemático da literatura [Trinkenreich et al., 2015] e as medidas identificadas no mapeamento e estavam em uso na organização em questão foram marcadas como "uso comprovado".

No presente artigo, é discutida uma extensão do trabalho descrito em [Trinkenreich e Santos, 2014] para avaliar também uma medida relacionada ao processo Orçamento e Contabilização de Serviços do MR-MPS-SV [Softex, 2012a] sob os dois aspectos também considerados em [Trinkenreich e Santos, 2014]: (i) se o processo é estável e capaz de atender ao indicador colocado como meta anual pela organização, sob a luz do Controle Estatístico de Processos [Rocha et al., 2012], e (ii) se a medida em uso pela organização é capaz de atender o objetivo de negócio, sob a luz do GQM [Solingen e Berghout, 1999]. Além disso, a seção de revisão da literatura foi estendida e a descrição experiência foi estruturada de forma mais detalhada. Como resultado, neste artigo os autores apresentam novas lições aprendidas com a experiência, sugerem novas medidas a serem usadas pela organização, desta vez encontradas no mapeamento sistemático [Trinkenreich et al., 2015] para atender mais diretamente os objetivos organizacionais relacionados às medidas avaliadas.

O objetivo deste trabalho faz parte de uma pesquisa mais abrangente sobre como identificar e compreender melhor as medidas a serem utilizadas em iniciativas de melhoria de processo de serviços de TI e manter o alinhamento das medidas com os objetivos de negócios da organização.

\section{Descrição da Experiência}

A Pesquisa-Ação é um método pós-positivista de pesquisa que mistura pesquisa e prática, inclui sempre dois objetivos simultâneos, de solucionar um problema e também contribuir para a ciência (Coughlan e Coughlan, 2002). É considerado como um método adequado para o estudo da tecnologia no contexto humano, colocando o pesquisador num papel participativo de colaboração à atividade que está sendo estudada na organização em questão. Diferente dos métodos positivistas que possuem como objetivo somente a geração de conhecimento, a Pesquisa-Ação pode ser descrita como um método póspositivistas por ser empírico, mas interpretativo; experimental, mas multivariado; e observacional, mas intervencionista (Baskerville e Wood-Harper, 1996). O ciclo de Pesquisa-Ação inclui três tipos de fases, uma fase preliminar para compreender o contexto e os objetivos; uma fase principal com ciclos de coleta, validação e análise de dados, e planejamento, execução e avaliação das ações; e uma fase central para monitorar todo o trabalho (Coughlan e Coughlan, 2002). Estas fases serão detalhadas durante a 
descrição da experiência.

\subsection{Fase Preliminar - Compreendendo o Contexto e os Objetivos}

Nesta fase da Pesquisa-Ação é preciso compreender o motivo de a ação ser necessária ou desejável, e quais são as forças econômicas, políticas, sociais e técnicas que estão impulsionando a ação (Coughlan e Coughlan, 2002).

A experiência relatada nesse artigo aconteceu no departamento de Tecnologia de Informação (TI) de uma empresa global de grande porte, que executa seus processos seguindo as práticas da biblioteca ITIL [OGC, 2011], mas não é avaliada em nenhum modelo de maturidade de software ou serviços. Esta organização conta com aproximadamente 80.000 usuários de TI, tem sede no Brasil, mas possui escritórios distribuídos nos cinco continentes. O negócio da empresa não é TI, mas está fortemente apoiado na infraestrutura e sistemas que a TI fornece em forma de serviços.

O objetivo desta experiência, seguindo o template do GQM [Solingen e Berghout, 1999], pode ser sumarizado como:

Analisar o processo de medição e as medidas utilizadas por uma organização provedora de serviços de TI, com o propósito de avaliar a conformidade do processo, os resultados, a estabilidade e a capacidade das medidas em atender ao indicador colocado como meta anual pela organização a qualidade das medidas utilizadas, com respeito ao atendimento dos objetivos estratégicos da organização e do ponto de vista dos resultados esperados pelo processo de Medição (MED) do modelo de maturidade de serviços MRMPS-SV [Softex, 2012a], do Controle Estatístico de Processos [Rocha et al., 2012] e do método GQM [Solingen e Berghout, 1999], no contexto de um ambiente real da indústria e considerando medidas relacionadas aos subprocessos GIN, GCD e OCS.

A motivação para esta experiência ocorreu devido ao grande esforço despendido pelos funcionários da área de Serviços de Tecnologia da Informação em realizar a medição para atender as metas criadas pela organização. Os objetivos são transformados em metas mensuráveis que os funcionários precisam alcançar anualmente, e o monitoramento das ações em execução para o alcance nestas metas é realizado mensalmente durante todo o período. As medidas são criadas e aplicadas para diferentes áreas, com objetivo de motivar o trabalho intra e entre equipes em prol da melhoria da qualidade do serviço como um todo. Desta forma, os membros das equipes não se preocupam apenas com os processos que as suas áreas atendem, mas também apoiam a execução de outras áreas. Além de metas de projetos e redução de custos, também existem metas relacionadas ao atendimento de incidentes dentro do prazo e ao tempo de disponibilidade de sistemas considerados críticos de alto impacto ao negócio. $\mathrm{O}$ escopo deste trabalho se limitará à avaliação do processo de medição para metas relacionadas a GIN (Percentual de incidentes resolvidos dentro do prazo acordado no ANS), GCD (Tempo total de indisponibilidade ao usuário em casos de crise - TTR Time to Repair) e OCS (Percentual de redução de custo com servidores). As três medidas foram selecionadas por serem aqueles que a empresa têm maior dificuldade de atender e porque os dados da organização que participou da pesquisa-ação estarem disponíveis ao pesquisador. Além disso, devido ao controle dos três processos ser sugerido por [Brooks, 2014], devido ao processo Orçamento e Contabilização de Serviços (OCS) não ter aparecido em nenhuma medida encontrada no mapeamento sistemático realizado pelos autores em [Trinkenreich et al., 2015] e devido ao relacionamento existente entre os 
processos Gerência de Incidentes e Gerência de Continuidade e Disponibilidade dos Serviços.

\subsection{Fase Principal}

\subsubsection{Coleta, Verificação e Análise de Dados}

Em uma pesquisa-ação, a coleta de dados pode ser realizada de diferentes formas, de acordo com o contexto. Análise de relatórios e documentações, entrevistas e discussões, ou até em observações durante conversas informais. A observação de comportamentos dentro da organização é uma fonte de coleta de dados relevante na Pesquisa-Ação, e pode contar com dinâmicas de grupos durante seu trabalho, como por exemplo padrões de comunicação em grupos, comportamentos de liderança, uso de poder, formas de solução de problemas e de tomadas de decisão (Coughlan e Coughlan, 2002). Nesta experiência, a coleta de dados foi realizada com base em entrevistas informais com pessoas selecionadas para obter informações sobre o processo de medição e as medidas em uso.

A validação dos dados é feita quando o pesquisador organiza os dados coletados de uma forma que seja possível validar e analisar as informações coletadas, podendo ser por meio de relatórios ou outras formas de apresentação (Coughlan e Coughlan, 2002). Nesta experiência, o processo de medição foi descrito e apresentado em termos dos resultados esperados de Medição (MED).

A análise dos dados é feita de forma colaborativa, em conjunto pelo pesquisador e pela organização, considerando que a organização conhece o seu negócio, sabe o que pode funcionar e será responsável pela execução das ações que forem definidas para serem executadas (Coughlan e Coughlan, 2002). Nesta experiência, o pesquisador introduziu para a organização os conceitos de estabilidade e capacidade de processos para avaliar se os processos associados pelas medidas em uso são estáveis e capazes de atender as metas definidas pela organização, sob a luz do Controle Estatístico de Processos.

Nesta experiência, a fase de coleta, validação e análise de dados foi composta por realização de entrevistas com as pessoas selecionadas para obter informações sobre o processo de medição e sobre as medidas em uso, além de descrição do processo de medição em termos dos resultados esperados de Medição (MED) e das etapas propostas pelo método GQM.

\subsubsection{Entrevistar pessoas selecionadas}

Um analista da equipe de qualidade foi entrevistado para explicar como as medidas são coletadas e analisadas. Analistas das equipes de Disponibilidade, Incidentes e Orçamento foram entrevistados para explicar o motivo da seleção das medidas em questão e a associação delas com os objetivos do negócio. O analista de qualidade possui conhecimento avançado sobre medição e algum conhecimento sobre as áreas de serviços de TI. Os analistas envolvidos não possuem o mesmo conhecimento em medição que o analista de qualidade, mas possuem profundo conhecimento sobre a área de serviços nas quais estão inseridos.

Para entender as medidas relacionadas a GIN e GCD é relevante conhecer o conceito de "Crise" e "Aplicações Críticas de Alto Impacto". Um incidente candidato a ser escalado para uma situação de "Crise" está relacionado a uma aplicação crítica de alto impacto. As aplicações classificadas desta forma impactam o negócio da organização 
caso seu nível de serviço seja alterado. Elas são divulgadas para o Service Desk saber como classificar o incidente caso ele chegue por um usuário ou por um evento de monitoramento. Um incidente sobre uma localidade inteira indisponível também é candidato a "Crise". Assim, os processos GIN e GCD estão relacionados. A assertividade na definição dos incidentes candidatos a crises permitem que o processo de escalação de incidentes seja mais preciso, aumentando a produtividade e apoiando na redução do tempo de avaliação dos resultados das medidas.

Cada servidor ligado na organização em questão representa custos fixos de suporte ao hardware, licenças de software, armazenamento de dados e backup. Visando ao objetivo estratégico de redução de custos, diversas ações e projetos de consolidação de servidores foram iniciadas, e o indicador, relacionado ao processo OCS, foi criado para quantificar o percentual de redução de custos atingido com o desligamento de servidores. Sempre consultando as áreas de negócio e avaliando com elas o risco e impacto de cada ação, os analistas das equipes de sistemas e infraestrutura trabalham juntos em projetos de consolidação. Sistemas que estavam instalados em servidores dedicados foram reavaliados quanto à necessidade de isolamento, sistemas legados que estavam sendo usados apenas para consulta foram exportados para bases de dados estáticas, ambientes não produtivos (homologação, testes, desenvolvimento etc.) foram reavaliados quanto à necessidade, entre outras iniciativas. Independente das ações tomadas, a forma de medição utilizada pela organização foi avaliar o percentual de redução de custo alcançado com desligamento de servidores.

\subsubsection{Descrever o processo de medição}

As medidas foram identificadas a partir dos objetivos estratégicos da organização, documentados na Tabela 2, seguindo a etapa de definição do GQM [Solingen e Berghout, 1999]. Desta forma, verificou-se que a Tabela 2 evidencia os resultados esperados MED1

- Objetivos de medição são estabelecidos e mantidos a partir dos objetivos de negócio da organização e das necessidades de informação de processos técnicos e gerenciais e MED2 - Um conjunto adequado de medidas, orientado pelos objetivos de medição, é identificado e definido, priorizado, documentado, revisado e, quando pertinente, atualizado.

Tabela 2. Objetivos, questões e medidas

\begin{tabular}{|c|c|c|c|c|c|}
\hline $\begin{array}{c}\text { Objetivos } \\
\text { Organizacionais }\end{array}$ & \begin{tabular}{|c|}
$\begin{array}{c}\text { Objetivo da } \\
\text { Medida }\end{array}$ \\
\end{tabular} & \begin{tabular}{|c|} 
Processo \\
Associado \\
\end{tabular} & Medida Base & $\begin{array}{c}\text { Medida } \\
\text { Derivada }\end{array}$ & Medida \\
\hline $\begin{array}{l}\text { Reduzir o tempo } \\
\text { de resolução de } \\
\text { incidentes }\end{array}$ & $\begin{array}{l}\text { Fornecer uma visão } \\
\text { sobre incidentes } \\
\text { atendidos no prazo }\end{array}$ & GIN & $\begin{array}{l}\text { MB1: Quantidade de } \\
\text { incidentes atendidos no prazo } \\
\text { MB2: Quantidade total de } \\
\text { incidentes atendidos }\end{array}$ & MB1/MB2 & $\begin{array}{l}\text { Incidentes } \\
\text { atendidos no } \\
\text { prazo (por } \\
\text { criticidade) }\end{array}$ \\
\hline $\begin{array}{l}\text { Reduzir tempo de } \\
\text { indisponibilidade } \\
\text { de aplicações } \\
\text { críticas }\end{array}$ & $\begin{array}{l}\text { Fornecer uma visão } \\
\text { sobre a efetividade de } \\
\text { resolução de crises }\end{array}$ & GCD & $\begin{array}{l}\text { MB3: Tempo para escalar } \\
\text { incidente a crise } \\
\text { MB4: Tempo total da crise }\end{array}$ & MB3+MB4 & $\begin{array}{l}\text { Diminuir tempo } \\
\text { total em crise }\end{array}$ \\
\hline $\begin{array}{l}\text { Reduzir custos } \\
\text { com servidores } \\
\text { em produção }\end{array}$ & $\begin{array}{l}\text { Fornecer uma visão } \\
\text { sobre o percentual de } \\
\text { redução de custos com } \\
\text { servidores desligados }\end{array}$ & OCS & $\begin{array}{l}\text { MB5: Custo dos servidores } \\
\text { eliminados } \\
\text { MB6: Custo total de } \\
\text { servidores }\end{array}$ & $\begin{array}{l}\text { (MB5/ } \\
\text { MB6) x100 }\end{array}$ & $\begin{array}{l}\text { Redução de custo } \\
\text { com servidores }\end{array}$ \\
\hline
\end{tabular}

Mensalmente, o analista de qualidade coleta os dados e armazena as medidas. Para o indicador "Diminuir tempo total em crise", ele recebe um relatório da equipe de crises e somente copia os valores para a planilha de medição que contém as fórmulas para 
análise do indicador. Para o indicador "Incidentes atendidos no prazo", ele executa uma busca no banco de dados do sistema de gerenciamento de incidentes para extrair a quantidade de incidentes atendidos no prazo e o total de incidentes atendidos. Para o indicador "Percentual de redução de custo com servidores", ele recebe a lista de servidores, com o respectivo registro de mudança de desligamento e os valores dos custos de hardware, licenças de software, armazenamento e backup de cada um dos gerentes de projetos. Os valores são exportados para a planilha de medição com fórmulas para análise do indicador. O procedimento sobre como coletar os dados é seguido e o armazenamento das medidas é feito, mas não está documentado. Portanto, o processo atual não atende ao MED3 - Os procedimentos para a coleta e o armazenamento de medidas são especificados. O plano de medição e análise para cada indicador contém a fonte da medida, a unidade, seu percentual da meta total (que é formada por um conjunto de medidas) sua fórmula de cálculo, seu objetivo, descrição e escopo, seus valores de referência e respectivas pontuações, conforme os planos de medição descritos nas Tabelas 3, 5 e 7. Assim, verificou-se que o processo atual atende ao resultado esperado MED4 Os procedimentos para a análise das medidas são especificados.

No início de cada ano as metas são revisadas e a coleta mensal se inicia. A periodicidade de apuração da meta é anual devido à política da empresa e porque o pagamento do bônus anual é atrelado ao alcance dela, mas a coleta e a medição são mensais. Os dados são coletados e avaliados (Tabelas 4, 6 e 8) segundo o plano de medição de cada medida (Tabelas 3, 5 e 7).

Os valores acumulados no ano de 2014 (56\% de incidentes atendidos no prazo, $1157 \mathrm{~h} 58 \mathrm{~min}$ em crise e $1,99 \%$ de redução de custos com servidores) são comparados com a meta $(89,84 \%$ de incidentes atendidos no prazo, $1500 \mathrm{~h}$ em crise e $7 \%$ de redução de custos com servidores) para analisar o desempenho do processo até o momento.

Foi verificado que duas das três metas ainda poderiam ser alcançadas. A meta de incidentes não pode mais ser recuperada, porque nos quatro meses de Setembro a Dezembro ela teria que atingir $158 \%$ para a média anual chegar em $89,84 \%$. A meta de tempo em crise pode ser alcançada caso o tempo médio nos quatro meses de Setembro a Dezembro fosse de até $85 \mathrm{~h} 30 \mathrm{~min}$. Apesar de ser muito difícil, a meta de servidores poderia ser alcançada caso os projetos dos quatro meses de Setembro a Dezembro reduzissem em média $1,25 \%$ de custo dos servidores por mês, o que representaria aproximadamente $\mathrm{R} \$ 59.000,00$ de redução de custo por mês.

Para chegar ao mínimo de $5 \%$ da meta, a redução mensal nestes quatro últimos meses deveria ser $0,75 \%$, o que representa aproximadamente $\mathrm{R} \$ 35.000,00$ de redução de custo por mês. Do ponto de vista de MED, o processo atende ao resultado esperado MED5 - Os dados requeridos são coletados e analisados.

Tabela 3. Plano de Medição para "Incidentes atendidos no prazo"

\begin{tabular}{|l|l|l|l|l|}
\hline $\begin{array}{l}\text { Indicador: Incidentes } \\
\text { atendidos no prazo }\end{array}$ & $\begin{array}{l}\text { Período de } \\
\text { Apuração: Anual }\end{array}$ & $\begin{array}{l}\text { Responsável pela medição: } \\
\text { Equipe de Qualidade }\end{array}$ & $\begin{array}{l}\text { Percentual } \\
\text { total: } 10,00 \%\end{array}$ \\
\hline Evidências: Relatórios gerados pela equipe de qualidade & Unidade de medida: $\%$ & Início: $01 / 01 / 2014$ \\
\hline Cálculo: Número de incidentes fechados dentro do ANS / Número incidentes fechados & Fim: $31 / 12 / 2014$ \\
\hline $\begin{array}{l}\text { Objetivo, descrição } \text { e escopo e fonte de apuração: Este indicador visa avaliar a eficiência para atender incidentes } \\
\text { Severidade 1 e 2 para aplicações críticas de alto impacto, fonte: base de dados de incidentes. }\end{array}$ \\
\hline Níveis esperados: & Mínimo: $85,36 \%$ & Alvo: $89,94 \%$ & Máximo: $92,53 \%$ \\
\hline Pontuação: & 100 & 125 \\
\hline
\end{tabular}


Tabela 4. Coleta de dados para "Incidentes atendidos no prazo"

\begin{tabular}{|c|c|c|c|}
\hline & $\begin{array}{c}\text { Total de incidentes atendidos } \\
\text { no prazo }\end{array}$ & $\begin{array}{c}\text { Total de incidentes } \\
\text { atendidos }\end{array}$ & $\begin{array}{c}\text { Percentual de incidentes atendido } \\
\text { no prazo }\end{array}$ \\
\hline Jan/2014 & 416 & 1038 & $40,08 \%$ \\
\hline Fev/2014 & 422 & 745 & $56,64 \%$ \\
\hline Mar/2014 & 378 & 654 & $57,80 \%$ \\
\hline Abr/2014 & 479 & 661 & $72,47 \%$ \\
\hline Mai/2014 & 411 & 741 & $55,47 \%$ \\
\hline Jun/2014 & 375 & 870 & $43,10 \%$ \\
\hline Jul/2014 & 451 & 759 & $59,42 \%$ \\
\hline Ago/2014 & 436 & 698 & $62,46 \%$ \\
\hline \multicolumn{2}{|r|}{ Total até Agosto/2014 } & & $\mathbf{5 5 , 9 3 \%}$ \\
\hline
\end{tabular}

Tabela 5. Plano de Medição para "Diminuir tempo em crise"

\begin{tabular}{|c|c|c|c|c|}
\hline $\begin{array}{l}\text { Indicador: Diminuir tempo } \\
\text { em crise }\end{array}$ & \begin{tabular}{|l|} 
Período de \\
Apuração: Anual
\end{tabular} & $\begin{array}{l}\text { Responsável pela medição: } \\
\text { Equipe de Qualidade }\end{array}$ & & $\begin{array}{lll}\text { entual } & \text { da } \quad \text { meta } \\
: 10,00 \% & \end{array}$ \\
\hline \multicolumn{2}{|c|}{ Evidências: Relatórios gerados pela equipe de crises } & \multicolumn{2}{|c|}{ Unidade de medida: Horas } & Início: $01 / 01 / 2014$ \\
\hline \multicolumn{4}{|c|}{ Cálculo: Tempo para escalar incidente a crise + Tempo total da crise } & Fim: $31 / 12 / 2014$ \\
\hline \multicolumn{5}{|c|}{$\begin{array}{l}\text { Objetivo, descrição e escopo e fonte de apuração: Reduzir o impacto ao negócio causado por indisponibilidade de } \\
\text { aplicações críticas de alto impacto. }\end{array}$} \\
\hline Níveis esperados: & Mínimo: 1900 horas & \multicolumn{2}{|c|}{ Alvo: 1500 horas } & Máximo: 1100 \\
\hline Pontuação: & 50 & \multicolumn{2}{|l|}{100} & 125 \\
\hline
\end{tabular}

Tabela 6. Coleta de dados para "Diminuir tempo em crise"

\begin{tabular}{|c|c|c|c|}
\hline & Tempo entre incidente e crise & Tempo em crise & Tempo total de indisponibilidade \\
\hline Jan/2014 & $26: 06: 00$ & $90: 51: 00$ & $116: 57: 00$ \\
\hline Fev/2014 & $24: 13: 00$ & $126: 59: 00$ & $151: 12: 00$ \\
\hline Mar/2014 & $14: 50: 00$ & $117: 28: 00$ & $132: 18: 00$ \\
\hline Abr/2014 & $11: 50: 00$ & $259: 22: 00$ & $271: 12: 00$ \\
\hline Mai/2014 & $1: 23: 00$ & $6: 48: 00$ & $8: 11: 00$ \\
\hline Jun/2014 & $30: 12: 00$ & $69: 11: 00$ & $99: 23: 00$ \\
\hline Jul/2014 & $27: 16: 00$ & $85: 25: 00$ & $112: 41: 00$ \\
\hline Ago/2014 & $48: 09: 00$ & $217: 55: 00$ & $266: 04: 00$ \\
\hline \multicolumn{2}{r|}{ Total até Agosto/2014 } & & $\mathbf{1 1 5 7 : 5 8 : 0 0}$ \\
\hline
\end{tabular}

Tabela 7. Plano de Medição para "Reduzir custo com servidores"

\begin{tabular}{|l|l|l|l|}
\hline $\begin{array}{l}\text { Indicador: Reduzir custo } \\
\text { com servidores }\end{array}$ & $\begin{array}{l}\text { Período de Apuração: } \\
\text { Anual }\end{array}$ & $\begin{array}{l}\text { Responsável pela medição: } \\
\text { Equipe de Qualidade }\end{array}$ & $\begin{array}{l}\text { Percentual da meta } \\
\text { total: } 15,00 \%\end{array}$ \\
\hline Evidências: Registros de mudanças & Unidade de medida: Percentual & Início: $01 / 01 / 2014$ \\
\hline $\begin{array}{l}\text { Cálculo: } \text { Redução de custos alcançadas com desligamento de servidores / Custo total dos } \\
\text { servidores em Janeiro/2014 }\end{array}$ & Fim: $31 / 12 / 2014$ \\
\hline Objetivo, descrição e escopo e fonte de apuração: Reduzir o custo com servidores em produção \\
\hline Níveis esperados: & Mínimo:5\% & Alvo:7\% & Máximo: $10 \%$ \\
\hline Pontuação: & 50 & 100 & 125 \\
\hline
\end{tabular}

Tabela 8. Coleta de dados para "Reduzir custo com servidores"

\begin{tabular}{|c|c|c|c|}
\hline & $\begin{array}{c}\text { Custo anual dos servidores } \\
\text { desligados }\end{array}$ & $\begin{array}{c}\text { Custo anual de todos os } \\
\text { servidores em Janeiro/2014 }\end{array}$ & $\begin{array}{c}\text { Redução de custo com } \\
\text { desligamento de servidores }\end{array}$ \\
\hline Jan/2014 & $\mathrm{R} \$ 942,37$ & $\mathrm{R} \$ 4.035 .316,84$ & $0,02 \%$ \\
\hline Fev/2014 & $\mathrm{R} \$ 11.137,5$ & $\mathrm{R} \$ 4.035 .316,84$ & $0,28 \%$ \\
\hline Mar/2014 & $\mathrm{R} \$ 6.521,44$ & $\mathrm{R} \$ 4.035 .316,84$ & $0,16 \%$ \\
\hline $\mathbf{A b r} / \mathbf{2 0 1 4}$ & $\mathrm{R} \$ 10.865,56$ & $\mathrm{R} \$ 4.035 .316,84$ & $0,27 \%$ \\
\hline Mai/2014 & $\mathrm{R} \$ 20.824,61$ & $\mathrm{R} \$ 4.035 .316,84$ & $0,52 \%$ \\
\hline $\mathbf{J u n} / \mathbf{2 0 1 4}$ & $\mathrm{R} \$ 2.321,89$ & $\mathrm{R} \$ 4.035 .316,84$ & $0,06 \%$ \\
\hline $\mathbf{J u l} / \mathbf{2 0 1 4}$ & $\mathrm{R} \$ 15.179,24$ & $\mathrm{R} \$ 4.035 .316,84$ & $0,38 \%$ \\
\hline $\mathbf{A g o} / \mathbf{2 0 1 4}$ & $\mathrm{R} \$ 12.666,46$ & $\mathrm{R} \$ 4.035 .316,84$ & $0,31 \%$ \\
\hline \multicolumn{2}{|c|}{ Total até Agosto/2014 } & & $\mathbf{1 , 9 9 \%}$ \\
\hline
\end{tabular}

TRINKENREICH, B.; SANTOS, G.

Avaliação do Processo de Medição para Serviços de TI em uma Empresa Global à Luz do MR-MPS-SV

iSys - Revista Brasileira de Sistemas de Informação, Rio de Janeiro, vol. 8, No. 2, p. 58-77, 2015 


\subsubsection{Planejar ações}

O planejamento de ações é realizado em conjunto entre o pesquisador e a organização, para definir o que precisa ser mudado, em quais áreas da organização, quais tipos de mudança são necessários, qual o suporte necessário para executar as mudanças, como o comprometimento com a mudança será estabelecido e como a resistência à mudança será gerenciada (Coughlan e Coughlan, 2002).

Nesta experiência, as mudanças planejadas abrangeram uma nova forma de avaliação do resultado da medição, melhorias para as medidas em uso e também para o processo de medição, conforme descrito a seguir.

\subsubsection{Utilizar uma nova forma de avaliar os resultados das medições}

O processo Medição não requer que sejam feitas predições por meio uma análise quantitativa e estatística como os níveis mais altos de maturidade (A e B) do MR-MPS$\mathrm{SV}$, mas para avaliar se os processos eram capazes de alcançar as metas estabelecidas, os processos foram analisados estatisticamente. Apesar de as Figuras 1 e 2 mostrarem que os processos relacionados a estas duas medidas eram estáveis, os intervalos entre os limites de controle de ambas as medidas estavam muito grandes, e não permitiriam atender efetivamente aos objetivos da organização ou tomar conclusões efetivas de previsibilidade sobre o comportamento futuro dos processos. No caso da medida "Reduzir custo com servidores" (Figura 3), observou-se que o valor apurado em Janeiro/2013 ficou fora dos limites de controle. Ao excluir os dois primeiros meses da análise, os limites de controle se estreitaram, mas mesmo assim percebe-se uma grande variação. A organização deveria investigar oportunidades de melhoria para diminuir estas variações, de forma a facilitar o planejamento da equipe e o esforço necessário para atender incidentes dentro do acordo de nível de serviços (ANS), diminuir o tempo de indisponibilidade de aplicações críticas de alto impacto e conseguir reduzir o custo com desligamento de servidores.

Os dados para medição e a situação atual de cada área são armazenadas em planilhas, mas estão em uma pasta compartilhada da rede cujo acesso é feito somente pelo analista de desempenho que realiza a coleta e a análise. Com isso, o resultado esperado MED6 - Os dados e os resultados das análises são armazenados é parcialmente atendido.

Existe uma reunião mensal de líderes, mas a análise da medição de cada indicador é discutida apenas trimestralmente, quando são avaliados os valores acumulados no ano até o momento, para avaliar a situação atual em relação ao alcance das metas. Nesta reunião são verificados os sistemas que mais influenciaram o indicador que estiver abaixo da meta e são criados planos de ações corretivas para melhoria. Entretanto, não há um local onde todos podem acessar diretamente e acompanhar os resultados das medições até o momento da escrita deste artigo. Desta forma, resultado esperado MED 7 - Os dados e os resultados das análises são comunicados aos interessados e são utilizados para apoiar decisões foi considerado parcialmente atendido. 

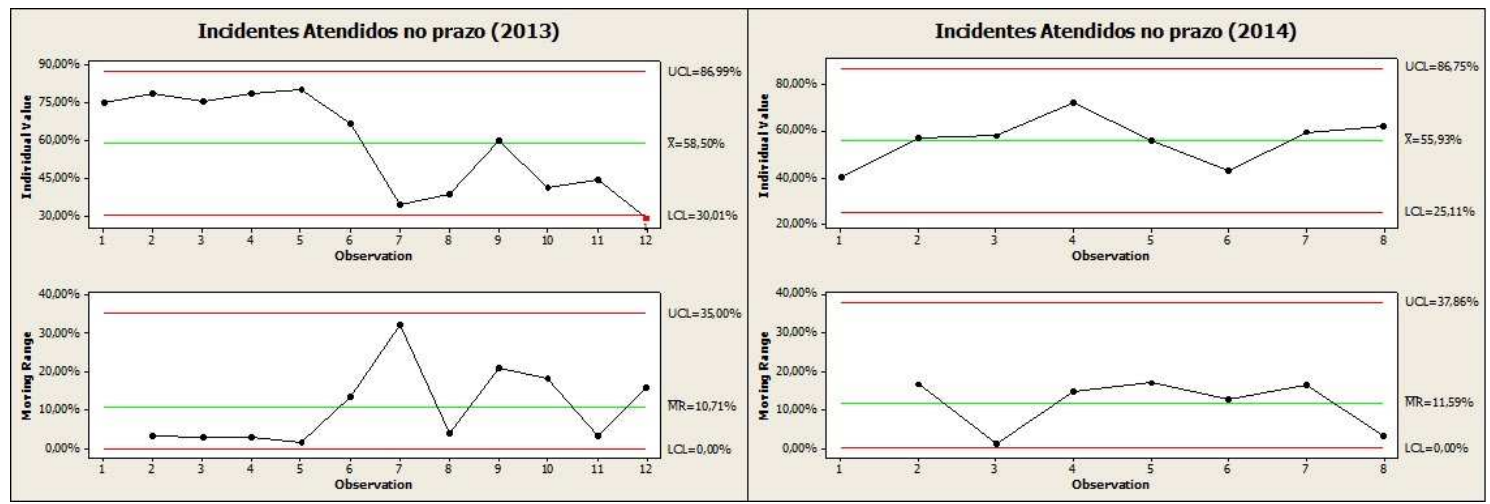

Figura 1- Gráficos de Controle para "Incidentes atendidos no prazo"
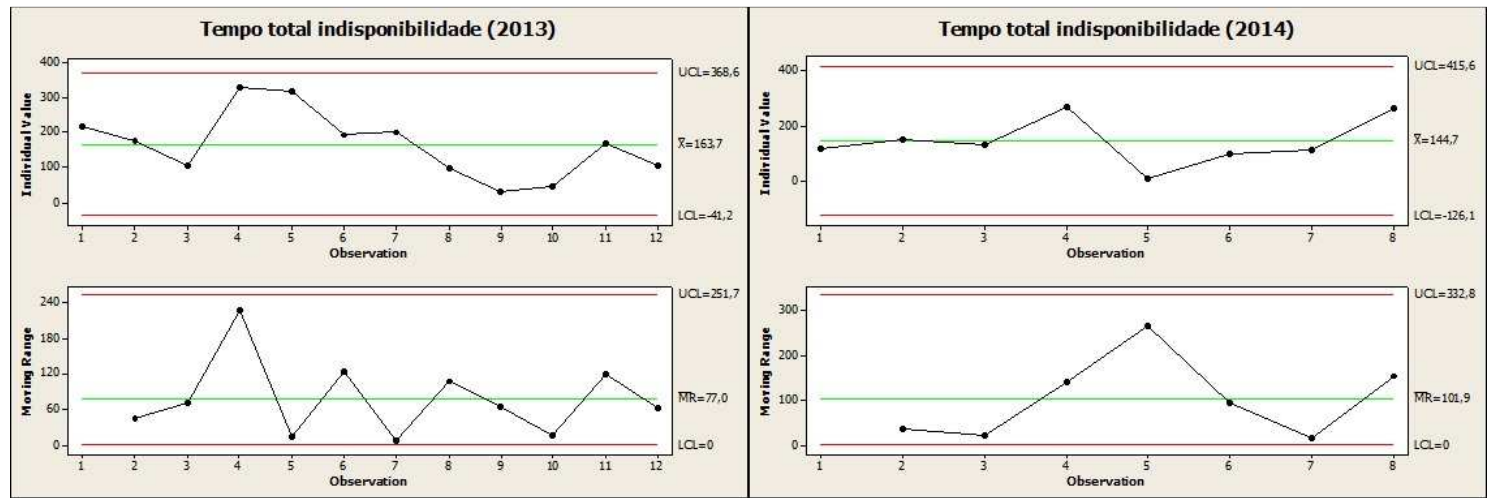

Figura 2 - Gráficos de Controle para "Diminuir tempo em crise"
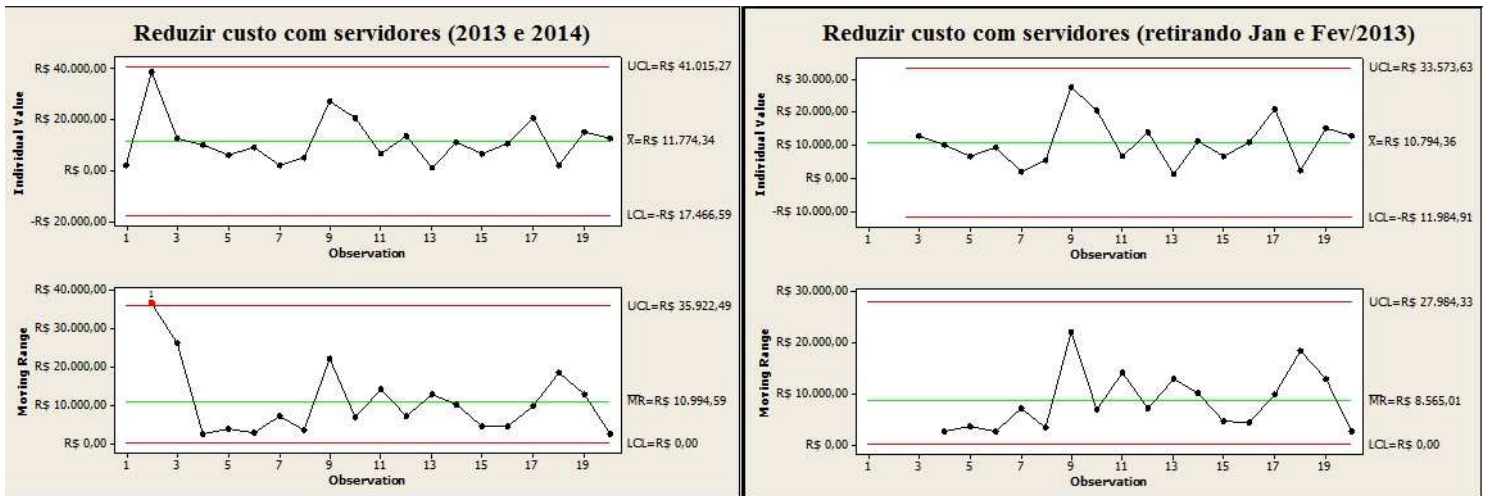

Figura 3 - Gráficos de Controle para "Reduzir Custo com Servidores"

\subsubsection{Implementar melhorias para as medidas em uso}

Foi observado que o indicador "Diminuir tempo total em crise" atende diretamente o processo GCD, mas é também associado a outros processos do MR-MPS-SV, como:

(i) GIN, pois um incidente é escalado à crise quando está impactando uma aplicação crítica de alto impacto e ferindo o ANS acordado entre a área de TI e o cliente;

(ii) GNS, pois o objetivo do indicador é reduzir ao máximo o tempo total de impacto ao usuário, visando atender o ANS em relação ao tempo de disponibilidade dos sistemas;

(iii) GPL, pois um registro de problema é sempre aberto após uma crise para investigação da causa raiz da falha. Observou-se também que o indicador "Incidentes atendidos no prazo" atende diretamente o processo GIN, mas é 
também associado ao processo GNS, pois o objetivo do indicador é atender os incidentes dentro do tempo acordado no ANS.

A qualidade das medidas pode ser melhorada. O indicador "Diminuir tempo total em crise" seria mais efetivo medindo diretamente o tempo de indisponibilidade do serviço, em crise ou não. O objetivo deste indicador é incentivar os envolvidos a fazerem o melhor possível para que a crise seja fechada o quanto antes, mas medir a indisponibilidade diretamente pela ferramenta de monitoramento teria a coleta de dados automatizada, menos passível a erro humano, mais barata e confiável. Outras medidas de avaliação da disponibilidade poderiam ser: Tempo médio para restaurar um serviço após falha e Tempo médio entre falhas de serviço [Lepmets et al., 2014]. Outras medidas relacionadas ao processo GCD descritas em [Trinkenreich et al., 2015] são: Impacto nos negócios causados por falhas no serviço de TI; Quantidade e duração de interrupções de serviço por mês, aplicação, item de configuração; Processos de negócios que possuem e que não possuem acordos de continuidade; Quantidade de práticas de recuperação de desastres por mês, por aplicação e item de configuração; Número de medidas preventivas de desastres implementadas. A organização planeja realizar uma mudança incluindo medições de disponibilidade que sejam trazidas diretamente de sistemas de monitoramento, mas continuar com a medição do tempo em crise.

O indicador "Incidentes atendidos no prazo" é observado na literatura sobre medição de qualidade de serviços de TI, mas outras medidas para avaliação do gerenciamento de incidentes poderiam ser usadas: Incidentes relacionados a desempenho, capacidade, continuidade, integridade, disponibilidade e confidencialidade de informação; Incidentes atendidos por dia [Lepmets et al., 2014]; Tempo de resolução dos incidentes fechados dentro/fora do ANS; Quantidade de incidentes repetidos, causados por execução de mudanças e que geraram abertura de problemas; Taxa de aumento de problemas em relação a incidentes [Liu et al., 2011]; Quantidade de incidentes por estado, abertos e resolvidos no mês [Valverde e Talla, 2014], entre outros. Outras medidas relacionadas ao processo GIN descritas em [Trinkenreich et al., 2015] são: Quantidade de incidentes evitados por dia; Média de tempo entre incidentes; Média de tempo para a restauração do serviço; Quantidade de incidentes escalados e redirecionados; Média de tempo para registrar um incidente por telefone e sistema; Média de tempo para categorizar, priorizar, começar a resolver um incidente; Tempo médio de resposta por nível de suporte; Percentual de descrição correta de incidente; Percentual de existência de documentação para primeiro nível de serviço. A organização ainda precisa avaliar quais os dados disponíveis para realizar uma mudança e selecionar de novas medidas.

O indicador "Percentual de redução de custo com servidores" não foi encontrada na literatura exatamente desta forma para servidores, mas a medição de percentual redução de custo efetivo de produção é sugerida para controle financeiro como a razão do custo no mês corrente sobre o custo no trimestre anterior [Brooks, 2014]. O indicador "Percentual de redução de custo com servidores" encontra-se diretamente alinhado com o objetivo estratégico da organização de redução de custos. Ele está associado ao processo OCS e sua forma de cálculo, criada pela organização, está completa (incluindo todos os custos inerentes aos servidores) e transparece exatamente o que se deseja medir e alcançar. A organização planeja realizar uma mudança para expandir seu escopo de medidas com outras medições de redução de custos de produção para caixas de correio e contratos de manutenção. 


\subsubsection{Implementar melhorias para o processo de medição}

A utilização da abordagem de avaliação do processo atual de medição à luz do modelo de maturidade MR-MPS-SV, mesmo sem pretensão de avaliação da empresa, poderá apoiar a melhoria do processo, que precisa documentar os diferentes processos de coleta e armazenamento de dados e comunicar mais objetivamente o resultado das medições. Esta verificação do processo será replicada a outras medidas relacionadas aos demais processos (Gerência de Mudanças, Capacidade, Trabalho etc.). As mudanças planejadas de melhoria são: criar uma área no Microsoft Sharepoint, que é a ferramenta de colaboração oficial da empresa, documentar formalmente o processo de coleta e armazenamento dos dados (MED3), armazenar a documentação e os dados de forma centralizada, organizada e independente de acesso de um único funcionário (MED6), e prover acesso ao resultado da medição, mostrando a situação até o momento (MED7).

As melhorias propostas ao processo de medição poderão apoiar a melhoria da qualidade dos serviços, uma vez que os gestores terão visibilidade constante do resultado que as medidas estão apresentando, sendo capazes de agir em melhorias de seus processos de serviços e logo observar se as ações surtiram efeito no resultado das medidas.

\subsubsection{Execução das Ações}

A execução das ações é realizada pela organização de acordo com o planejamento realizado na atividade anterior. As mudanças planejadas na atividade anterior ainda estão em andamento, sendo executadas sob a forma de projetos pela organização.

Não foi necessário realizar nenhuma contratação ou contar com custos adicionais para as mudanças planejadas. Para as mudanças no processo, a área compartilhada no Microsoft Sharepoint foi criada e o processo de coleta e armazenamento dos dados está sendo documentado para possibilitar uma análise mais profunda dos planos de medição e iniciar a melhoria das medidas. A criação de uma área compartilhada para prover acesso ao resultado da medição, mostrando a situação até o momento ainda não foi iniciada pela organização.

\subsubsection{Avaliação das Ações}

A avaliação envolve a reflexão sobre os resultados de todo o processo de Pesquisa-Ação, para que o próximo ciclo se beneficie das lições aprendidas no ciclo atual, e das próprias ações, intencionais e não intencionais.

A Tabela 9 apresenta as lições aprendidas pelo pesquisador e pela organização durante esta experiência.

Tabela 9. Lições Aprendidas

\begin{tabular}{|c|c|}
\hline \# & Lição Aprendida \\
\hline 1 & $\begin{array}{l}\text { A avaliação da qualidade de serviços de TI pode ser feita em empresas, mesmo quando o negócio } \\
\text { não é TI, por meio da medição e alcance das metas de serviços. }\end{array}$ \\
\hline 2 & $\begin{array}{l}\text { A utilização de medidas relacionadas a mais de uma área de processo faz com que as equipes } \\
\text { diferentes trabalhem juntas e apoiem-se mutuamente para atingir o resultado esperado. }\end{array}$ \\
\hline 3 & $\begin{array}{l}\text { A empresa consegue fomentar o trabalho entre membros das equipes ao condicionar o resultado } \\
\text { individual ao resultado do grupo. Por exemplo, o analista de suporte da produção responsável pelo } \\
\text { sistema X pode ter os incidentes de seu sistema atendidos no prazo, mas ele precisa que o analista } \\
\text { da sua equipe, responsável pelo sistema Y também tenha os incidentes de seu sistema atendidos no } \\
\text { prazo, para alcançar a meta da equipe que ambos fazem parte. }\end{array}$ \\
\hline 4 & A empresa consegue fomentar o trabalho entre diferentes equipes, ao utilizar, mesmo com pesos \\
\hline
\end{tabular}




\begin{tabular}{|c|l|}
\hline$\#$ & \multicolumn{1}{c|}{ Lição Aprendida } \\
\hline & $\begin{array}{l}\text { diferentes, a medida de uma equipe para a outra. Por exemplo, o gerente de projetos pode ter 20\% } \\
\text { de sua meta total relacionada à entrega do projeto no prazo. Para contar com o apoio da produção, } \\
\text { a meta total da equipe de produção também conta com o indicador de entrega de projetos no prazo, } \\
\text { mesmo que o percentual seja menor, de 5\%. }\end{array}$ \\
\hline 5 & $\begin{array}{l}\text { A prática de criação de metas mensuráveis para a área de TI pode ser considerada por outras } \\
\text { empresas e a formação de metas totais que considerem indicadores "intra equipes" (entre os } \\
\text { membros da mesma equipe) e entre equipes, além da criação de medidas que sozinhas já permeiem } \\
\text { mais de uma equipe (exemplo: Incidentes causados por mudanças). }\end{array}$ \\
\hline 6 & $\begin{array}{l}\text { Metas mensuráveis de redução de custos para a área de TI encorajam atividades de consolidação de } \\
\text { infraestrutura, simplificação de processos e sistemas e diminuição de exigências de altos níveis de } \\
\text { serviço por parte dos usuários para ambientes que não são realmente críticos ao negócio. }\end{array}$ \\
\hline 7 & $\begin{array}{l}\text { Mesmo sendo possível encontrar medidas relacionadas aos processos de serviços na literatura, não } \\
\text { é trivial avaliá-las no contexto real de uma organização, devido à falta de dados disponíveis e, } \\
\text { quando disponíveis, grande variação nos limites de controle para testes estatísticos. }\end{array}$ \\
\hline
\end{tabular}

Esta experiência possui questões que podem limitar a validade dos resultados [Wohlin et al., 2012]. Como ameaça de validade interna, para a análise do processo, as informações de caracterização que o participante forneceu de si mesmo (ele é o executor) foram utilizadas, e não é possível confirmar que tais informações fornecidas estejam corretas. Como ameaça de validade externa, pode-se citar que o processo de mapeamento em etapas pode ser replicado para outras empresas, mas a avaliação do processo e das medidas, e também as conclusões apresentadas, são particulares para cada caso. Como ameaças de validade de constructo, (i) pode existir algum viés no relato do analista de qualidade em entrevista aberta, visando esconder ou minimizar falhas no processo seguido por ele; e (ii) foram selecionadas somente três medidas em uso pela organização. Como cada medida possui um processo de medição diferente, outras medidas podem demandar alterações de processo que não foram mapeadas neste estudo.

\subsection{Fase de Monitoramento}

O monitoramento é uma meta-fase que acontece durante todos os ciclos do processo de Pesquisa-Ação. Cada ciclo do processo de Pesquisa-Ação leva o início de um novo ciclo com planejamento, execução e avaliação sendo realizados de forma contínua, criando oportunidade de aprendizado também contínuo (Coughlan e Coughlan, 2002).

Durante o monitoramento da experiência, foi verificado que o processo de seleção de medidas de serviços de TI pode incluir uma fase de mapeamento das integrações entre os processos para inclusão de medidas correlacionando mais de um processo, e medidas de desempenho distribuídas entre diferentes equipes para estimular o trabalho e o apoio entre diferentes equipes.

\section{Considerações Finais}

Este artigo apresentou uma avaliação sobre a medição nos processos de Gerência de Incidentes, Gerência de Continuidade e Disponibilidade de Serviços e Orçamento e Contabilização de Serviços de uma empresa global de grande porte, sob à luz do processo Medição do modelo de maturidade MS-MPS-SV. Verificou-se que as medidas são corretamente derivadas dos objetivos estratégicos da organização, mas que o processo de medição e as próprias medidas podem ser ampliados para atenderem melhor os objetivos da empresa.

Como ciclos futuros para a Pesquisa-Ação, serão avaliados, sob a luz do MR- 
MPS-SV (Softex, 2012a), os próprios processos de serviços GCD, GIN e OCS na organização, para verificar como eles podem ser melhorados e como a melhoria reflete no resultado de suas medidas. Além disso, as demais medidas usadas pela empresa serão estudadas, assim como a correlação entre os processos de serviços e a estabilidade e capacidade de processos associados às medidas para encontrar aquelas que podem ser sugeridas para aplicação do Controle Estatístico de Processos (CEP) [Florac e Carleton, 1997], apoiando a organização na previsão do comportamento futuro dos processos e com isso avaliar se estará apta a atender suas metas definidas.

\section{Agradecimentos}

Os autores agradecem à FAPERJ pelo auxílio financeiro (projeto E-26/110.438/2014).

\section{Referências}

Baskerville, R. L., Wood-Harper, A. T. (1996) "A critical perspective on action research as a method for information systems research" Journal of Information Technology, vol. 11, pp. 235-246.

Brooks, P. (2014) -“Metrics for service management: designing for ITIL"- Van Haren Publishing, Zaltbommel, www.vanharen.net.

Coughlan, P., Coughlan, D. (2002) "Action research for operations management" International Journal of Operations \& Production Management. vol. 22 pp. 220-240.

Forrester, E., Buteau, B., Shrum, S. (2010) - CMMI for Services, Guidelines for Superior Service. CMMI-SVC Version 1.3 - 2nd Edition. SEI. Addison-Wesley Professional.

Demarco, T. (1982). Controlling software projects, Prentice-Hall, 1982.

Florac e Carleton (1997) "Practical Software Measurement: Measuring for Process Management and Improvement" Technical Report CMU/SEI-97-HB-003, Software Engineering Institute, Carnegie Mellon University, Pittsburgh, PA.

International Organization for Standardization/ International Eletrotechnical Commision (2003) "ISO/IEC 15504-2: Information Technology - Performing an Assessment".

International Organization for Standardization/ International Eletrotechnical Commision (2011) "ISO/IEC 20000 Information Technology - Service Management".

Jäntti, M., Lahtela, A., Kaukola, J. (2010) "Establishing a Measurement System for IT Service Management Processes: A Case Study". Int'l Journal on Advances in Systems and Measurements, vol. 3 no $3 \& 4$.

OGC, 2011, "ITIL Service Operations" The Stationary Office - TSO. London, UK

Parasuraman, A. Zeithaml, L. Berry, 1985. "A conceptual model of service quality and its implications for future research" Journal of Marketing, vol. 49, pp. 41-50.

Recker, J. (2013) "Scientific Research in Information Systems A Beginner's Guide". Springer, ISBN 978-3-642-30048-6.

Rocha, A. R., Santos, G., Barcellos, M. P. (2012) - "Medição de Software e Controle Estatístico de Processos", Série de Livros PBQP, Editora MCT.

Softex (2012a) Associação para Promoção da Excelência do Software Brasileiro "MPS.BR - Guia Geral MPS de Serviços". Disponível em www.softex.br/mpsbr.

Softex (2012b) - Associação para Promoção da Excelência do Software Brasileiro "MPS.BR Guia Geral MPS de Software". Disponível em www.softex.br/mpsbr.

Softex (2012c) Associação para Promoção da Excelência do Software Brasileiro - "Guia de Implementação - Parte 2: Fundamentação para Implementação do Nível F do MR-MPSSV:2012". Disponível em www.softex.br/mpsbr.

Solingen, R., Berghout, E. (1999) "The Goal/Question/Metric Method: A Practical Guide for Quality Improvement of Software Development". McGraw-Hill.

Trinkenreich, B., Santos, G. (2014) "Avaliação do Processo de Medição em Gerência de Incidentes e Gerência de Continuidade e Disponibilidade à Luz do MR-MPS-SV". $10^{\circ}$

TRINKENREICH, B.; SANTOS, G.

Avaliação do Processo de Medição para Serviços de TI em uma Empresa Global à Luz do MR-MPS-SV

iSys - Revista Brasileira de Sistemas de Informação, Rio de Janeiro, vol. 8, No. 2, p. 58-77, 2015 
Workshop Anual do MPS (WAMPS), Campinas, SP.

Trinkenreich, B., Santos, G., Barcellos, M. P. (2015) "Metrics to Support IT Service Maturity Models - A Systematic Mapping Study", 17th International Conference on Enterprise Information Systems (ICEIS), Barcelona, Espanha.

Trinkenreich, B., Santos, G. (2015) "Metrics to Support IT Service Maturity Models - A Case Study", 17th International Conference on Enterprise Information Systems (ICEIS), Barcelona, Espanha.

Lepmets, M., Mesquida, A. L., Cater-Steel, A., Mas, A., Ras, E. (2014) "The Evaluation of the IT Service Quality Measurement Framework in Industry", Global Journal of Flexible Systems Management.

Liu, M., Gao, Z., Luo, W., \& Wan, J. (2011) "Case Study on IT Service Management Process Evaluation Framework Based on ITIL" Business Management Electronic Information Conference, vol. 2, pp. 199-202, Guangzhou, China, IEEE.

Valverde, R., \& Talla, M. (2014) "DSS Based IT Service Support Process Reengineering Using ITIL: A Case Study" Journal Intelligent Decision Technologies vol. 55 pp. 35-65 Springer Berlin Heidelberg.

Wohlin, C., Runeson, P., Höst, M., Regnell, B., Wesslén, A. (2012) "Experimentation in Software Engineering" Springer Science \& Business Media 236pp ISBN-13: 978-3642290435. 